\title{
Chemical defenses of the tropical ascidian Atapozoa sp. and its nudibranch predators Nembrotha spp.
}

\author{
Valerie J. Paul ${ }^{1}$, Niels Lindquist ${ }^{2}$, William Fenical ${ }^{2}$ \\ ${ }^{1}$ University of Guam Marine Laboratory, UOG Station, Mangilao, Guam 96923, USA \\ ${ }^{2}$ Scripps Institution of Oceanography, University of California San Diego, La Jolla, California 92093-0228, USA
}

\begin{abstract}
The ascidian Atapozoa sp. is common in shallow reef habitats throughout the tropical IndoPacific. Collections from the central Philippine Islands, northern Sulawesi (Indonesia), Palau, Kwajalein, and Ant Atoll (near Ponape) were examined for the presence of secondary metabolites. In most cases, nudibranch molluscs Nembrotha spp. were found feeding on the ascidians. Like many dorid nudibranchs, these appear to obtain their defensive chemicals through their diet. Both ascidians and nudibranchs contained large quantities of a series of bipyrrole secondary metabolites previously described as the tambjamines. Concentrations of the different tambjamines and a related, highly bluepigmented tetrapyrrole varied among collections of the ascidians and nudibranchs. Overall, concentrations of tambjamines and the tetrapyrrole were higher in the nudibranchs, and were the major components of the mucus exuded by Nembrotha spp. When irritated. Tambjamine A and the tetrapyrrole were present in the nudibranchs but did not occur in detectable dmounts in Atapozoa. The ascidian and nudibranch extracts and isolated metabolites were tested as feeding deterrents toward a variety of carnivorous fishes in field assays on 2 Guam reefs that differed in the types of fishes that fed during the assays. The crude extract, mixtures of tambjamines, tambjamine $C$, tambjamine $F$, and the tetrapyrrole were all significant feeding deterrents at or below natural concentrations. Tambjamines $A$ and $E$ were not deterrent when tested alone at natural concentrations; however, a 1:1 mixture of tambjamines $\mathrm{E}$ and $\mathrm{F}$ was deterrent when tested below natural concentrations. Evidence is thus presented that both the ascidian and its nudibranch predators use the tambjamines as chemical defenses against predators.
\end{abstract}

\section{INTRODUCTION}

Ascidians are a rich source of bioactive secondary metabolites that show cytotoxic, antimicrobial, and antiviral activities (Ireland et al. 1988). Over $100 \mathrm{com}$ pounds, primarily alkaloids or amino acid-derived metabolites, have been isolated and chemically defined (Faulkner 1984, 1986, 1987, Fenical 1986, Ireland et al. 1988). Secondary metabolites from marine invertebrates have been proposed as chemical defenses toward predators, pathogens, and fouling organisms (Bakus 1981, Bakus et al. 1986, Coll \& Sammarco in press); however, few studies have experimentally tested the defensive functions of these compounds (Coll et al. 1982, Thompson et al. 1982, 1985, Gerhart 1984, Bingham \& Braithwaite 1986, La Barre et al. 1986, Pawlik et al. 1986, 1987, 1988, Harvell et al. 1988, Paul \& Van Alstyne 1988, Wylie \& Paul 1989). Feeding deterrent functions of ascidian secondary metabolites have not been examined.
Many benthic ascidians have been proposed to suffer relatively little predation by generalist predators (Millar 1971, Goodbody \& Gibson 1974, Stoecker 1980c). High vanadium concentrations, low $\mathrm{pH}$, and secondary metabolites have all been proposed as defenses against predation and fouling in some species (Stoecker 1978, 1980a, b, c); however, Parry (1984) proposes that neither $\mathrm{pH}$ nor the presence of vanadium prevents predation on ascidians. In the tropics, some angelfishes, spadefishes, filefishes, and pufferfishes are known to consume relatively large amounts of ascidians (Randall \& Hartman 1968, Myers 1983). Molluscs such as Trochus niloticus and Turbo spp. have been observed to feed on a variety of ascidians on the Great Barrier Reef (Parry 1984). Specialized predators on ascidians include molluscs such as the lamellarians, cypraeids (cowries), and nudibranchs, and polyclad flatworms (Millar 1971, Morris et al. 1980, Parry 1984). On the other hand, some evidence for chemical defenses in ascidians has been observed. The larvae of the 
ascidians Didemnum molle and Ecteinascidia turbinata were rejected by fish predators (Olson 1983, Young \& Bingham 1987). Larvae of E. turbinata seemed to be defended by low molecular weight organic metabolites, although no deterrent secondary metabolites were actually isolated (Young \& Bingham 1987).

We commonly encountered the ascidian Atapozoa $\mathrm{sp}$. in shallow reef habitats throughout tropical areas of the western Pacific. The ascidians were abundant in the central Philippines, northern Sulawesi in Indonesia, Palau, Kwajalein Atoll, and Ant Atoll near Ponape (Fig. 1). They were not encountered on reefs surrounding the islands of Ponape, Yap, Guam, or the northern Mariana Islands. Atapozoa sp. was frequently grazed by nembrothid nudibranchs, including Nembrotha cristata and N. kubaryana, but we did not observe predation on the ascidian by generalist predators such as fishes or molluscs. Preliminary chemical studies of the extracts of both ascidians and nudibranchs showed that they contained large amounts of nitrogenous secondary metabolites known as tambjamines (Carte \& Faulkner 1983, 1986). The broad distribution of Atapozoa and its close association with nudibranchs of the genus Nembrotha allowed us to address questions about the chemical defenses of these organisms: (1) Does Atapozoa produce secondary metabolites and do these compounds function as chemical defenses toward generalist predators? (2) How do the chemistry and feeding deterrent effects of the nudibranchs compare with the host ascidian? (3) How do concentrations of secondary metabolites vary among collections of the ascidian and nudibranchs?

\section{MATERIALS AND METHODS}

Collections. Collections of Atapozoa sp, were made in shallow water (usually $<10 \mathrm{~m}$ deep) habitats in several areas of the western Pacific (Fig. 1). Ascidians were collected from Legan Island in Kwajalein Atoll, Ant Atoll near Ponape, Palau, Bunaken Island near Manado in Sulawesi, and from several islands in the central Philippines. The ascidians were most abundant in areas of high current or surge. After being collected, they were either frozen or immediately placed in organic solvents (methanol and/or dichloromethane). Nudibranchs of the genus Nembrotha, including $N$. cristata, N. kubaryana and several unidentified species, were commonly observed grazing on the ascidians. These nudibranchs were also collected and frozen or stored in acetone or methanol.

Several collections were made of the exuded mucus of Nembrotha spp. from the Philippines. Nudibranchs were irritated by handling and the slimy blue-green mucus that was exuded was collected on filter paper. The mucus was dissolved in acetone, and the acetonesoluble extract was filtered and evaporated under reduced vacuum.

Chemical extraction and analyses. Ascidians were ground in a blender with acetone. The ground ascidians were filtered and extracted twice again with acetone, followed by extraction with methanol $(3 \times)$, and then the combined extracts were evaporated under vacuum leaving an oily organic residue. Nudibranchs were soaked in acetone at least 3 times, and the

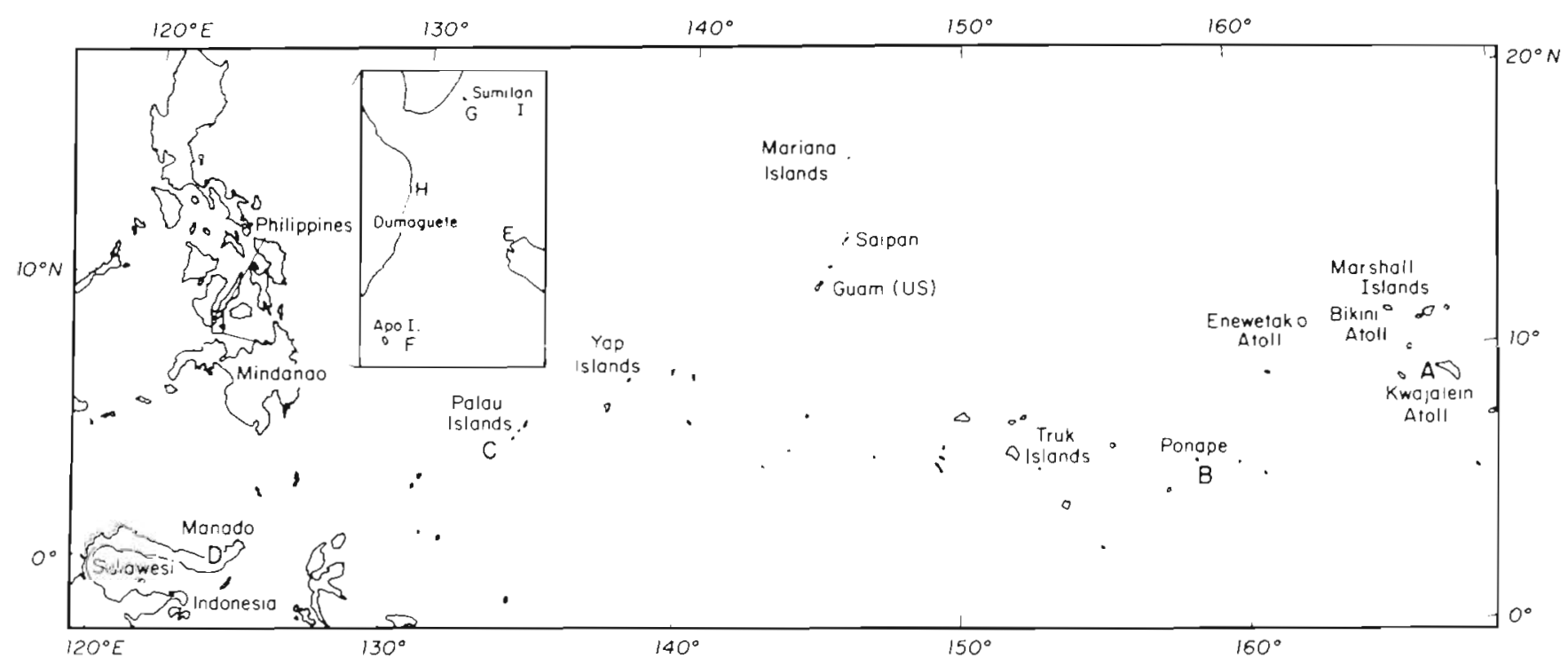

Fig. 1 The western Pacific indicating the collecting sites of Atapozod sp. and Nembrotha spp. Collecting sites were: A, Legan Island on the leeward side of Kwajalein Atoll; B. Ant Atoll near Ponape; C, rock islands in Palau; D. Bunaken Island near Manado. Indonesid; E. Siguijor Island, Philippines; F, Apo Island, Philippines; G, Sumilon Island, Philippines; H, Sillıman University Marine Lab. Dumaguete, Philippines 
acetone was evaporated under vacuum to leave an oily extract. Lipid-soluble extract yields were calculated as the dry weight of the extract divided by the dry weight of the extracted ascidian or nudibranch.

Identification of the secondary metabolites was accomplished by proton nuclear magnetic resonance (NMR) spectroscopy. Spectral values of the isolated metabolites were compared to published values in the literature to identify known compounds. Several metabolites were closely related to known metabolites but have not been previously reported. The structures of these new tambjamines have recently been determined (Lindquist \& Fenical unpubl.)

Concentrations of the different tambjamines were determined by isolating the metabolites by flashcolumn silica gel chromatography (using vacuum) and reverse-phase high performance liquid chromatography (HPLC). A solvent gradient from $100 \%$ dichloromethane to $1: 1$ methanol: dichloromethane was used to initially fractionate the extracts by silica gel flash chromatography. Tambjamines C, E, and F were further purified with a second silica gel flash column by using a finer gradient of the same solvents. Final purification was obtained by $\mathrm{C}-18$ reverse-phase HPLC with a solvent mixture of $8: 2$ methanol: water with $0: 1 \mathrm{M}$ ammonium acetate buffer. Tambjamine $\mathrm{A}$ was isolated by passing a column fraction from the initial fractionation of the crude extract of Nembrotha sp. through a Sephadex LH-20 column with $100 \%$ methanol as the eluting solvent. The tetrapyrrole was obtained in large enough quantities for field assays by flash column chromatography of the combined fractions that possessed this compound using hexane-ether solvent mixtures. For some collections, proton NMR analysis of fractions containing the tambjamines was used to estimate the quantity of different tambjamines present by integrating various peaks corresponding to different. tambjamines.

Feeding deterrent effects toward generalist carnivores. Field bioassays were conducted at 5 to $8 \mathrm{~m}$ depth at Fingers Reef in Apra Harbor and at Haps Reef in Agat Bay, Guam, USA. Many herbivorous and carnivorous fishes inhabit both reefs. The reef slope at Fingers is composed of scleractinian corals; Porites rus is especially common. Fishes observed to feed during experiments at Fingers Reef included damselfishes Abudefduf spp., wrasses Cheilinus fasciatus, Gomphosus varius, Halichoeres trimaculatus, Thalassoma lutescens, the emperor Lethrinus harak, and the triggerfish Balistapus undulatus. Haps Reef faces the open ocean on the western side of Guam. The top of the patch reef is composed of mostly dead Porites corals. Fishes observed to feed during experiments at Haps Reef included mostly triggerfishes Melichthys vidua, Balistapus undulatus and occasionally damselfishes
Dascyllus trimaculatus and Abudefduf spp. Thus, we tested the compounds toward 2 different populations of predatory reef fishes since the species that fed from the ropes differed at the 2 sites. These fishes are common throughout reef habitats of the western Pacific (Amesbury \& Myers 1982, Myers 1989) and represent a variety of potential generalist predators of Atapozoa and Nembrotha.

Thin strips of squid (ca $3 \mathrm{~mm}$ thick, $1.5 \mathrm{~cm} \times 1.5 \mathrm{~cm}$ square, dried with paper towels) were injected and coated with extracts or purified metabolites dissolved in $1: 1$ diethyl ether: acetone. Extracts, isolated metabolites, and mixtures of metabolites were tested at different concentrations representing the range of natural concentrations found in Atapozoa and Nembrotha. Control squid strips were treated with equal volumes of solvent only. When highly colored extracts or metabolites such as the tetrapyrrole were tested, the control solvent was dyed with blue or green food coloring so that treated and control squid would visually match. Solvents were allowed to evaporate from both treated and control pieces before being offered to fishes. Since the extracts and isolated metabolites (with the exception of tambjamine aldehyde) were all lipid-soluble, they adhere to the squid after the solvent evaporates and can then be placed in seawater. Similar methods have been used for testing feeding deterrent effects of seaweed and invertebrate secondary metabolites (see Hay \& Fenical 1988, Wylie \& Paul in press).

Four treated and 4 control pieces of squid were attached by paper clips to 2 separate yellow polypropylene lines. These lines were attached to the reef as a matched pair $(0.25$ to $0.5 \mathrm{~m}$ apart). Ten to 15 replicate pairs were used in each trial, and each pair was placed several meters apart on the reef. When at least 4 pieces of squid were consumed from the pair, the numbers of control and treated pieces eaten were recorded. Trials lasted only ca 15 to $20 \mathrm{~min}$ and compounds remained on the squid throughout the experiments as confirmed visually and by thin layer chromatography (TLC) after the assays. It is possible that some material was lost in seawater during the experiments; however, any losses of compounds would make our results more conservative. The numbers of squid pieces eaten on control and treated ropes were statistically compared by the Wilcoxon SignedRanks Test for paired comparisons. Parametric statistical procedures were not appropriate for some of our assays because the differences among pairs in the number of control and treated squid pieces eaten were not normally distributed. Since the Wilcoxon Test for paired comparisons is usually conservative when compared to the paired-sample $t$-test, we chose to use it for all of the statistical analyses (Zar 1984). 


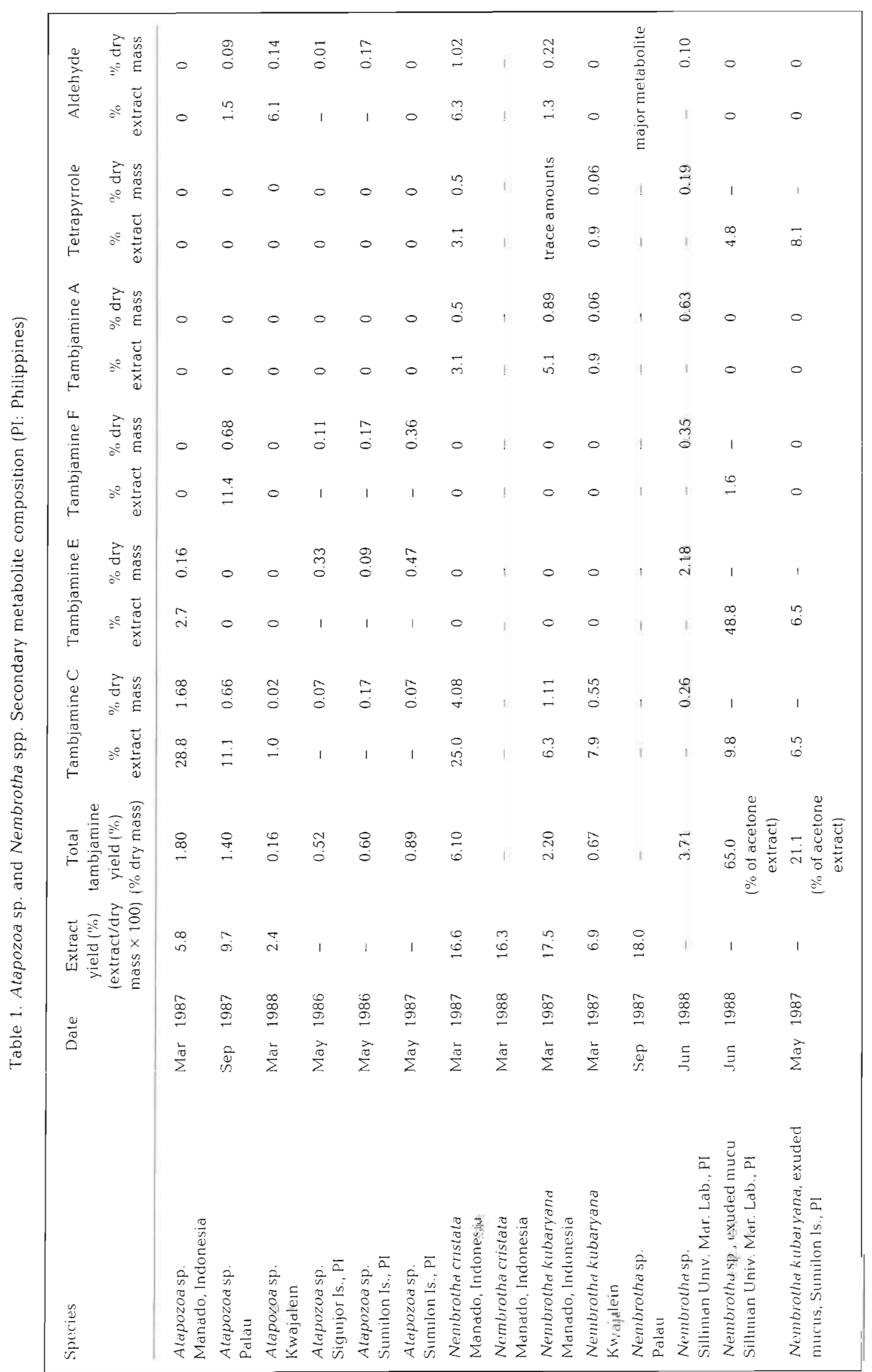




\section{RESULTS}

Similar secondary metabolites were found in all collections of Atapozoa sp.; however, the concentrations of different metabolites varied among collections for both the ascidian and the nudibranchs (Table 1). Atapozoa sp. contained a series of bipyrroles known as the tambjamines (Fig. 2) that have been previously isolated from the bryozoan Sessibugula translucens, its nudibranch predators Tambja abdere and Tambja eliora, and the nudibranch Roboastra tigris that preys on Tambja spp. from the Gulf of California (Carte \& Faulkner 1983, 1986). Tambjamines have also been found in the Indo-Pacific bryozoan Bugula dentata (Lindquist \& Fenical unpubl.). The tambjamines found in collections of Atapozoa sp. included tambjamines C, $E$, and $F$ (Table 1; Fig. 2). Only tambjamine $C$ has been previously described (Carte \& Faulkner 1983). None of the Atapozoa tambjamines were halogenated, although both bryozoans $S$. translucens and $B$. dentata contained halogenated tambjamines (Carte \& Faulkner 1983, Lindquist \& Fenical unpubl.). Some collections of Atapozoa also contained tambjamine aldehyde which has been shown to be a hydrolysis product of the other tambjamines and therefore an artifact of isolation (Carte \& Faulkner 1983). None of the ascidian collec-
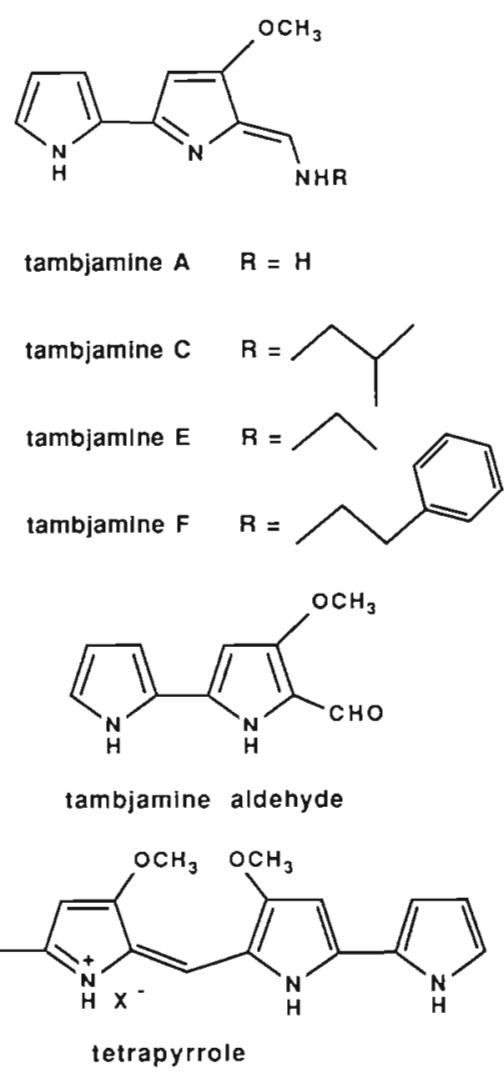

Fig. 2. Structures of the major metabolites isolated from collections of Atapozoa sp. and Nembrotha spp. tions contained quantifiable amounts of tambjamine A or the tetrapyrrole (Table 1), although small amounts of the tetrapyrrole were often detectable by thin layer chromatography (TLC).

Since some of the collections were handled differently (frozen versus extracted immediately), and some tambjamines are unstable and may hydrolyze to tambjamine aldehyde, small quantitative differences in the concentrations of different tambjamines among collections may not be significant. Nonetheless, some patterns of distribution of the tambjamines are readily apparent in Table 1. Overall, concentrations of crude organic extracts and total tambjamines were higher in the nudibranchs Nembrotha spp. than in Atapozoa sp. Generally, the presence of the different tambjamines in collections of Nembrotha resembled the patterns found in Atapozoa from the same habitats. For example, collections of Atapozoa and Nembrotha from Manado (Indonesia) and Kwajalein contained high amounts of tambjamine $C$ but very low or undetectable concentrations of tambjamines $E$ and F. Philippines collections of both organisms had high concentrations of tambjamines $C, E$, and $F$ (Table 1). In addition, extracts of all collections of Nembrotha contained tambjamine $\mathrm{A}$ and the tetrapyrrole which were not detected in Atapozoa (Table 1; Fig. 2). Both metabolites have been previously described; tambjamine A was isolated from Sessibugula translucens (Carte \& Faulkner 1983) and the tetrapyrrole was isolated from an undescribed Western Australian ascidian, the bryozoan Bugula dentata, and a mutant strain of the bacterium Serratia marcescens (Kazlauskas et al. 1982, Matsunaga et al. 1986). Tambjamine aldehyde was also present in some extracts of Nembrotha, but this is a hydrolysis product and likely results from breakdown of the other tambjamines.

Several collections of Atapozoa sp. and Nembrotha spp. were made that were not quantified because they were extracted in the field; however, the tambjamines were identified and their relative concentrations determined. Of the tambjamines isolated from Atapozoa sp. from Ant Atoll, tambjamine C accounted for $66.4 \%$ of the total tambjamines, tambjamine $\mathrm{E}$ was $20.2 \%$, tambjamine $\mathrm{F}$ was $2.2 \%$, and the aldehyde was $11.2 \%$. From Nembrotha cristata from Ant Atoll, tambjamine A accounted for $39 \%$ of total tambjamines, tambjamine C was $39 \%$, tambjamine E was $5.5 \%$, the aldehyde was $11 \%$, and the tetrapyrrole was $5.5 \%$. From Nembrotha kubaryana from Sumilon Island, Philippines, tambjamine $\mathrm{C}$ accounted for $30.8 \%$ of total tambjamines, tambjamine $E$ was $30.8 \%$, and the tetrapyrrole was $38.4 \%$. From Nembrotha sp. from Apo Islands, Philippines, tambjamine $\mathrm{C}$ accounted for $11.8 \%$ of total tambjamines, tambjamine $\mathrm{E}$ was $47 \%$, tambjamine $\mathrm{F}$ was $5.9 \%$, and tambjamine aldehyde was $35.3 \%$. 
These relative concentrations are similar to those reported in Table 1 since Atapozoa contained tambjamines $C, E$, and $F$ and Nembrotha spp. contained very low or undetectable amounts of tambjamine $F$ and relatively high concentrations of the tetrapyrrole.

The exuded mucus of Nembrotha contained high concentrations of the tambjamines as well as of the tetrapyrrole. When the nudibranchs were initially handled the exudate was dark blue, resembling the color of the isolated tetrapyrrole. This dark blue color was also released into seawater if the nudibranchs were prodded or attacked. The exudate changed to green upon continual handling. Tambjamines $F$ and $A$, which were present in the extracts of the whole nudibranchs, were in low or undetectable concentrations in the mucus. The tetrapyrrole was present in relatively high concentrations in the exuded mucus.

The crude extract of Atapozoa from Ant Atoll was a significant feeding deterrent $(\mathrm{p}=0.05)$ and reduced feeding by $24 \%$ relative to controls at a concentration of $2 \%$ dry weight, which is below natural concentrations (Fig. 3). Feeding deterrent effects of extracts of Atapozoa and Nembrotha kubaryana from Kwajalein were compared at equal concentrations of $1 \%$ of squid dry weight. The Nembrotha extract was more deterrent but results were not significant at the low concentrations used in the assay $(0.1>\mathrm{p}>0.05,2$-tailed test;

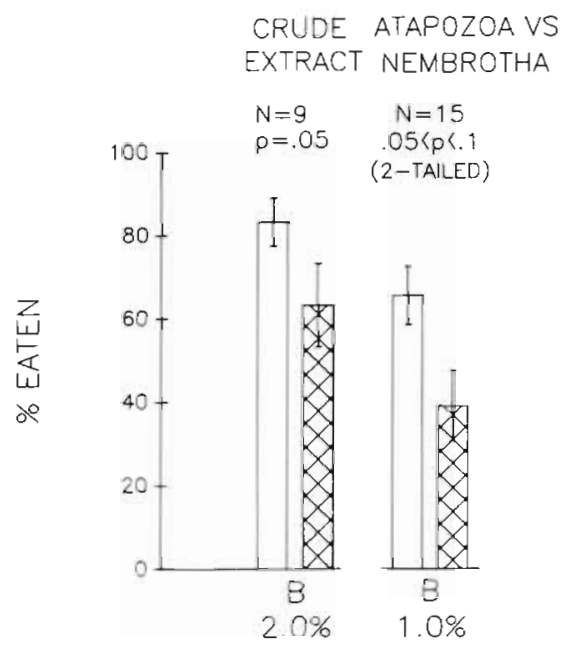

Fig. 3. Results of field assays with crude extracts of Atapozoa and Nembrotha kubaryana expressed as \% of squid pieces completely consumed ( $\bar{X} \pm 1 S E)$. Left: Crude extract of Atapozoa obtaned from Ant Atoll collections and tested at $2 \%$ of squid dry weight which is below natural concentrations. Open bar control; cross-hatch: treated. Right: Deterrent effects of Atapozoa sp. and Nembrotha kubaryana from Kwajalein Atoll compared at equal concentrations of $1 \%$ dry weight. Open bar. Atapozoa extract; cross-hatch: Nembrotha extract. (B: Assays conducted at Fingers Reef, Guam.) Results were analyzed by the Wilcoxon Signed-Ranks Test for paired comparisons (1-tailed test unless otherwise noted)
Fig. 3). Natural concentration of the Atapozoa extract from Kwajalein was $2.4 \%$ of dry weight; however, Nembrotha extracts occurred at much higher concentrations of $6.9 \%$ of dry weight (Table 1 ).

Results of field assays with isolated tambjamines and mixtures of tambjamines on 2 Guam reefs are shown in Fig. 4. Tambjamine $C$ was a major metabolite in most collections of Atapozoa and Nembrotha spp. (Table 1). It was a significant feeding deterrent on both reefs at concentrations as low as 0.4 to $0.5 \%$ dry weight, which is within the range of natural concentrations for Atapozoa and below natural concentrations for Nembrotha. Tambjamine F was a significant feeding deterrent at Haps Reef at $0.6 \%$ dry weight $(p<0.004$, grazing reduced $71.7 \%$ relative to controls) but not at $0.4 \%$ dry weight (Fig. 4). Again, these concentrations fall within the range of natural concentrations for tambjamine F. Tambjamine E was not a significant feeding deterrent on either reef at concentrations of up to $1.2 \%$ of dry weight, which is well above natural concentrations (Fig. 4). Tambjamine A was not deterrent at $0.2 \%$ of dry weight, and the aldehyde was not deterrent at $1.0 \%$ dry weight; both were tested within their range of natural concentrations. The most deterrent metabolite that we tested was the tetrapyrrole, which was effective at $0.1 \% \mathrm{dry}$ weight. This concentration was within the range that occurs naturally in the nudibranchs and is much lower than concentrations found in the mucus. All of the mixtures of tambjamines $\mathrm{C}, \mathrm{E}$, and $\mathrm{F}$ were deterrent at $0.5 \%$ dry weight and were as effective as pure tambjamine $C$. Mixtures of tambjamines $E$ and $F$ were deterrent; tambjamine $E$ alone was not.

\section{DISCUSSION}

The tambjamines were effective chemical defenses against generalist carnivorous fishes in field assays on Guam. These secondary metabolites are produced by the ascidian Atapozoa sp. in a variety of habitats in the western Pacific, as well as by several species of bryozoans. In addition, several species of nudibranchs of the genus Nembrotha feed on Atapozoa and appear to obtain the tambjamines from their diet in relatively high concentrations. Thus, these compounds serve as chemical defenses for the nudibranchs as well. These observations agree with the feeding deterrent effects previously reported for tambjamines from the bryozoan Sessibugula translucens and its nembrothid nudibranch predators. Mixtures of halogenated and nonhalogenated tambjamines isolated from the bryozoan and nudibranchs were deterrent toward the California spotted kelpfish Gibbonsia elegans in aquarium assays (Carte \& Faulkner 1986). 

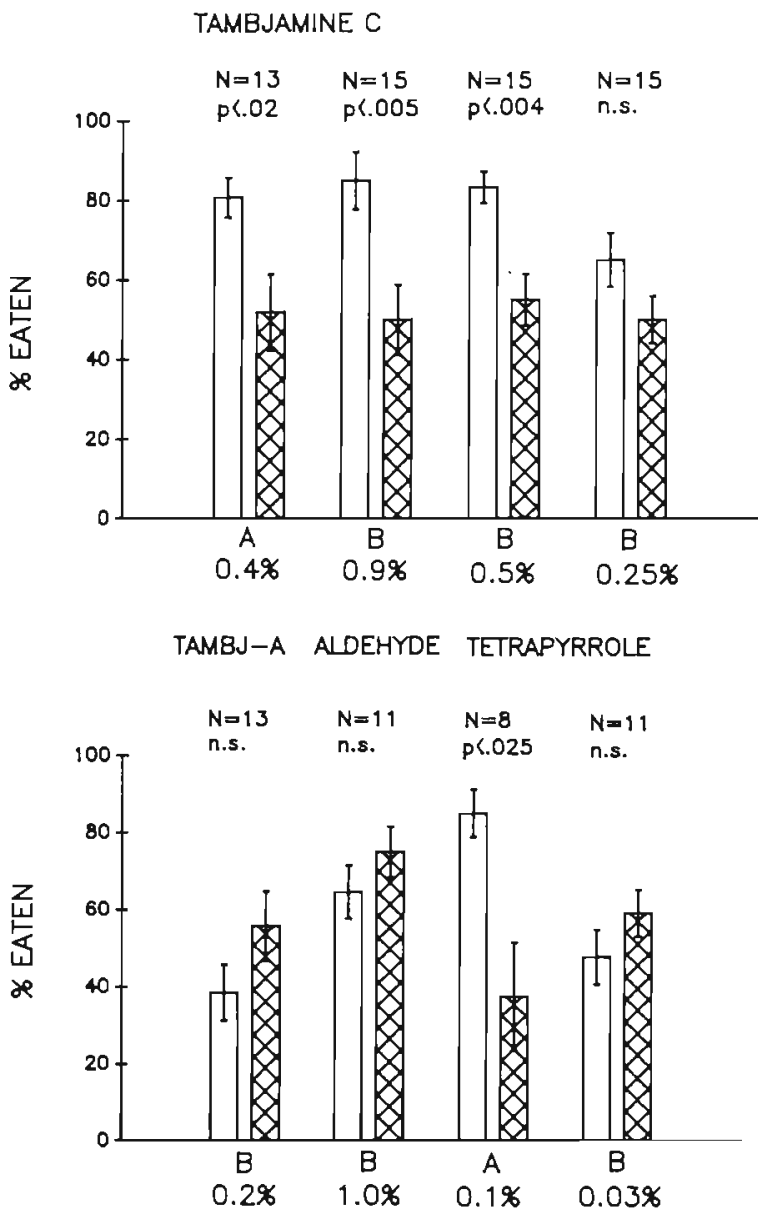

TAMBJAMINE F TAMBJAMINE E.
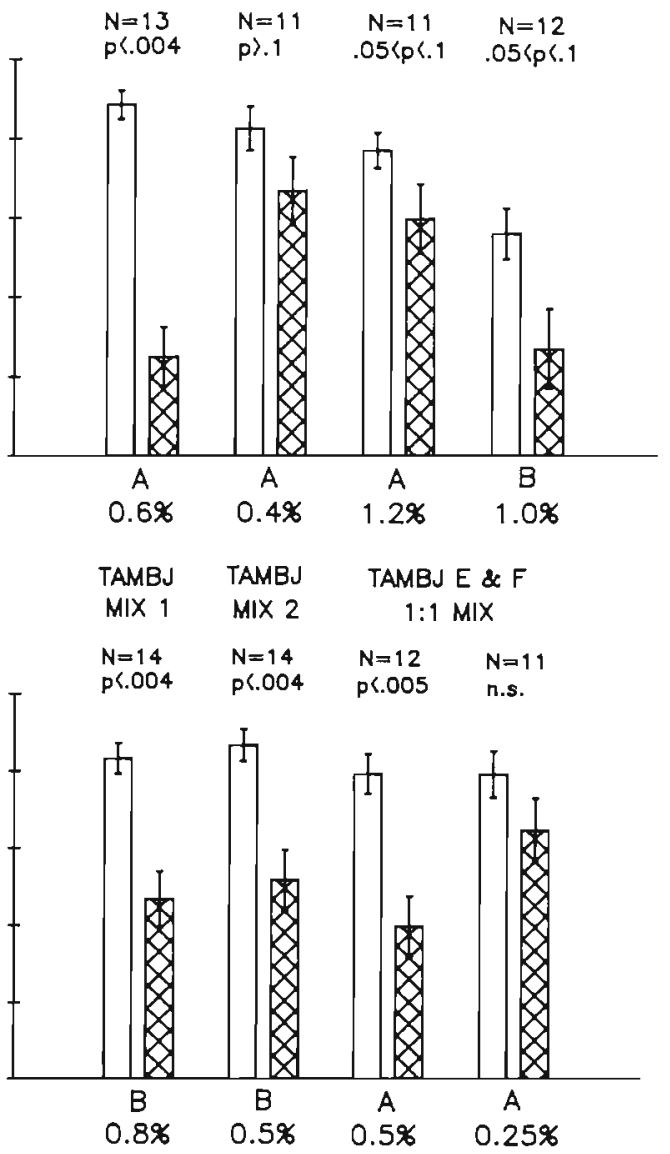

Fig. 4. Results of field assays with isolated tambjamines and mixtures of tambjamines on 2 Guam reefs expressed as \% of squid pieces completely consumed ( $\overline{\mathrm{X}} \pm 1 \mathrm{SE}$ ). A: Assays conducted at Haps Reef; $\mathrm{B}$ : Assays conducted at Fingers Reef; open bars: control squid; cross-hatch: treated squid. Concentrations represent \% dry weight of squid. Tambj Mix $1=2: 1$ tambjamine C: tambjamine F. Tambj Mix $2=6: 2: 1$ tambjamine E: tambjamine F: tambjamine C. Data were analyzed by the Wilcoxon SignedRanks Test for paired comparisons (1-tailed)

Based on our field assays, not all of the tambjamines were equally deterrent. Tambjamine $\mathrm{C}$ waş the most deterrent and was effective at a concentration of $0.4 \%$ dry weight. Tambjamine $\mathrm{C}$ was a major metabolite in most collections of the ascidian and nudibranchs. Tambjamine $\mathrm{F}$ was deterrent at $0.6 \%$ dry weight, but not at $0.4 \%$, and was found as a major metabolite in some collections of Atapozoa. Tambjamine $\mathrm{E}$ was not deterrent even at the relatively high concentration of $1.2 \%$ dry weight; however, $1: 1$ mixtures of tambjamines $\mathrm{E}$ and $\mathrm{F}$ were deterrent at $0.5 \%$ dry weight. In fact, all of the mixtures of tambjamines $C, E$, and $F$ that were tested were as effective as any single tambjamine tested alone. Most collections of Atapozoa contained a mixture of tambjamines including the most deterrent one, tambjamine C. Tambjamine aldehyde, which is probably not a natural product, was not effective at $1.0 \%$ dry weight. However, we had some problems testing this metabolite because, unlike the other metabolites, it was partially water-soluble and may have dissolved into seawater during the assays.
The most deterrent metabolite that we tested was the tetrapyrrole that occurs mainly in the nudibranchs. The tetrapyrrole is one of the major metabolites found in the mucus exuded by Nembrotha spp. when they are disturbed. The deep blue color of the tetrapyrrole may be a warning to potential predators when it is released into seawater. Since we reduced the effect of color by dying the control pieces of squid blue to match the treated pieces, the deterrent results were based only on the taste of this metabolite coated on the squid. The other metabolite found in Nembrotha spp. but not in Atapozoa sp. was tambjamine A. This metabolite was not deterrent at $0.2 \%$ dry weight; however, we did not have enough of the isolated metabolite to test it at its higher natural concentrations of 0.5 to $0.8 \%$ dry weight. Based on our chemical analyses and the assay comparing the deterrent effects of Atapozoa and Nembrotha, it appears that the nudibranchs are better defended chemically. Not only do they contain higher concentrations of the tambjamines, but they also contain more of the highly deterrent tetrapyrrole. 
Our observations on the relationship between Atapozoa sp. and its nudibranch predators support certain hypotheses about the chemical adaptations of dorid nudibranchs. Faulkner \& Ghiselin (1983) hypothesized that the evolution of shell-less opisthobranch mollusks such as the dorid nudibranchs was correlated with the presence of defense mechanisms based on chemicals derived from food. Dietary-derived chemicals seem to be a general method of defense for many different opisthobranch molluscs including sea hares (Faulkner 1988, Hay \& Fenical 1988), ascoglossans (Paul \& Van Alstyne 1988), and nudibranchs (Karuso 1987, Faulkner 1988, Pawlik et al. 1988). In the example we have described, the nudibranchs appear to either modify or selectively sequester some of the secondary metabolites found in their host ascidian. Both the deterrent tetrapyrrole and tambjamine $\mathrm{A}$ are present in relatively high concentrations in the nudibranchs but not in Atapozoa. Unlike many nudibranch predators, Nembrotha spp. are not aposematically colored. These nudibranchs are the same olive green color as Atapozoa sp. and are relatively difficult to see on their host.

Although the chemistry of many nudibranchs has been investigated, and many are known to contain high concentrations of secondary metabolites (see reviews by Faulkner \& Ghiselin 1983, Faulkner 1984 , Karuso 1987), few studies have actually investigated the defensive roles of these metabolites. In many cases, the diets of the nudibranchs are not known and can only be guessed based upon similarities between the chemicals found in the nudibranchs and in other invertebrates such as sponges (Faulkner 1984). Only a few studies have used potential natural predators to examine the effectiveness of nudibranch secondary metabolites as chemical defenses (Cimino et al. 1982, Thompson et al. 1982, Carte \& Faulkner 1986, Pawlik et al. 1988). This study is the first to demonstrate the effectiveness of dietary-derived chemical defenses in a nudibranch that feeds on ascidians.

Few other studies have linked secondary metabolites found in ascidians to those found in their predators. Marine prosobranch molluscs of the family Lamellariidae are known to feed on ascidians (Millar 1971). The tropical species Lamellaria sp. has been found to contain secondary metabolites that are similar to those produced by the tropical ascidian Didemnum chartaceum (Andersen et al. 1985, Lindquist et al. 1988).

The field assays used in these studies have both advantages and disadvantages when compared to those utilizing an artificial agar or carrageenan-based diet (e.g. Harvell et al. 1988). A major advantage is that many carnivorous fishes readily consume the squid pieces in the field and will immediately attack both treated and control pieces. Major disadvantages are that compounds cannot be evenly distributed throughout the food and water-soluble extracts or metabolites cannot be tested in these assays. We tried to minimize the first problem by using thin strips of squid which maximized the surface-to-volume ratio of the pieces and by both injecting and coating the metabolites on the squid. In support of these methods, many nudibranchs have higher concentrations of metabolites in the dorsal mantle (Thompson et al. 1982, Pawlik et al. 1988) and exude metabolites when they are irritated; thus, concentrations of metabolites are not evenly distributed throughout the animals. Many sponges also have higher concentrations of secondary metabolites in the surface layers than in the internal portions (Paul unpubl.). The only metabolite we tested that dissolved in seawater was tambjamine aldehyde. The other compounds were lipophilic and adhered to the squid.

It is interesting that identical or closely related tambjamines are produced by different phyla of invertebrates including bryozoans and ascidians. Nembrothid nudibranchs seem to specialize on prey items that contain these compounds. Tambja spp. feed on Sessibugula translucens in the Gulf of California (Carte \& Faulkner 1983, 1986), Nembrotha sp. feeds on Bugula dentata in Palau and Ponape (pers. obs.), and N. cristata, N. kubaryana, and Nembrotha sp. feed on Atapozoa sp. Because the tetrapyrrole is produced by bacteria as well as bryozoa and ascidians, and is related to bacterial pigments such as prodigiosin (Kazlauskas et al. 1982, Matsunaga et al. 1986), it has been suggested that the compound may be biosynthesized by bacteria associated with bryozoans or derived from food sources such as prodigiosin-producing bacteria (Matsunaga et al. 1986).

In conclusion, our data show that Atapozoa sp. and the associated predators Nembrotha spp. occur commonly throughout the tropical western Pacific. These animals contain several related tambjamines and concentrations of these metabolites vary among collections from different habitats. We did not analyze for individual or population variation within habitats; however, it appears that most naturally occurring combinations of the tambjamines are effective feeding deterrents toward generalist predatory coral-reef fishes. Tambjamines $\mathrm{C}$ as well as combinations of tambjamines $C, E$, and $F$ were all effective feeding deterrents at concentrations below natural levels. Nembrotha spp. contained high concentrations of the tetrapyrrole which was the most deterrent metabolite we tested. To the best of our knowledge, this study is the first to demonstrate chemical similarities between an ascidian and its specialist predators and to experimentally evaluate and compare the chemical defenses of nudibranchs and their host ascidian. 
Acknowiedgements. This research resulted from financial support by the National Institutes of Health (GM 38624 to V. J.P.) and the National Science Foundation (CHE86-20217 to W.F. and a predoctoral fellowshup to N. L.). We thank Dr Francoise Monniot, National Museum of Natural History, Paris, France for identifying Atapozoa sp. and Clay Carlson for identifying the nudibranchs. We are grateful to J. Faulkner, S. Nelson, J. Pawlik, K. Van Alstyne and 2 anonymous reviewers who commented on earlier drafts of this manuscript, and to $\mathrm{H}$ Sanger for assistance with the figures. This is contribution No. 274 of the University of Guam Marine Laboratory.

\section{LITERATURE CITED}

Amesbury, S. S., Myers, R. F. (1982). Guide to the coastal resources of Guam: Vol. 1. The fishes. University of Guam Press, Mangilao

Andersen, R. J., Faulkner, D. J., Cun-heng, H., Van Duyne G. D. Clardy, J. (1985). Metabolites of the marine prosobranch Lamellaria sp. J. Am. Chem. Soc. 107: 5492-5495

Bakus, G. J. (1981). Chemical defense mechanisms on the Great Barrier Reef. Science 211: 497-499

Bakus, G. J., Targett, N. M., Schulte, B. (1986). Chemical ecology of marine organisms: an overview. J. chem. Ecol. 12: 951-987

Bingham, B. L., Braithwaite, L. F. (1986). Defense adaptations of the dendrochirote holothurian Psolus chitonoides Clark. J. exp. mar. Biol. Ecol. 98: 311-322

Carte, B., Faulkner, D. J. (1983). Defensive metabolites from three nembrothid nudibranchs. J. org. Chem. 48: 2314-2318

Carte, B., Faulkner, D. J. (1986). Role of secondary metabolites in feeding associations between a predatory nudibranch, two grazing nudibranchs, and a bryozoan. J. chem. Ecol. 12: 795-804

Cimino, G., De Rosa, S., De Stefano, S., Sodano, G. (1982). The chemical defense of four Mediterranean nudibranchs. Comp. Biochem. Physiol. (B) 73: 471-474

Coll, J. C., La Barre, S. Sammarco, P. W. Williams, W. T., Bakus, G. J. (1982). Chemical defences in soft corals (Coelenterata: Octocorallia) of the Great Barrier Reef: a study of comparative toxicities. Mar Ecol. Prog. Ser 8: 271-278

Coll, J. C., Sammarco, P. W (in press). The role of secondary metabolites in the chemical ecology of marine invertebrates: a meeting ground for biologists and chemists. Proc. 6 th Internat. Coral Reef Symposium

Faulkner, D. J. (1984). Marine natural products: metabolites of marine invertebrates. Nat. Product Rep. 1. 551-598

Faulkner, D. J. (1986). Marine natural products. Nat. Product Rep. 3: 1-33

Faulkner, D. J. (1987). Marine natural products. Nat. Product Rep. 4: $539-576$

Faulkner, D. J. (1988). Feeding deterrents in mollusks. In: Fautin, D. G. (ed.) Biomedical importance of marine organisms. Mem. Calif. Acad. Sci. 13: 29-36

Faulkner, D. J., Ghiselin, M. T (1983). Chemical defense and evolutionary ecology of dorid nudibranchs and some other opisthobranch gastropods. Mar Ecol. Prog. Ser. 13: 295-301

Fenical, W. (1986). Marine alkaloids and related compounds. In: Pelletier, S. W. (ed.) Alkaloids: chemical and biological perspectives, Vol. 4. John Wiley \& Sons, Inc., New York, p. $275-330$

Gerhart, D. J. (1984). Prostaglandin $\mathrm{A}_{2}$ : an agent of chemical defense in the Caribbean gorgonian Plexaura homomalla. Mar. Ecol. Prog. Ser. 19: 181-187
Goodbody, I., Gibson, J. (1974). The biology of Ascidia nigra (Savigny) V Survival in populations settled at different times of the year. Biol. Bull. mar. biol. Lab., Woods Hole 146: $217-237$

Harvell, C. D., Fenical, W., Greene, C. H. (1988). Chemical and structural defenses of Caribbean gorgonians (Pseudopterogorgia spp.) I: Development of an in situ feeding assay. Mar. Ecol. Prog. Ser. 49: 287-294

Hay, M. E., Fenical, W. (1988). Marine plant-herbivore interactions: the ecology of chemical defense. Ann. Rev. Ecol. Syst. 19: 111-145

Ireland, C. M., Roll, D. M., Molinski, T F., McKee, T C. Zabriskie, T. M., Swersey, J. C. (1988). Uniqueness of the marine chemical environment: categories of marine natural products from invertebrates. In: Fautin, D. G. (ed.) Biomedical importance of marine organisms. Mem. Calif. Acad. Sci. 13: 41-57

Karuso, P. (1987). Chemical ecology of the nudibranchs. In: Scheuer, P. J. (ed.) Bioorganic marine chemistry. SpringerVerlag, Berlin, p 31-60

Kazlauskas, R., Marwood, J. F., Murphy, P. T., Wells, R. J. (1982). A blue pigment from a compound ascidian. Aust. J. Chem. 35: 215-217

La Barre, S. C., Coll, J. C., Sammarco, P. W. (1986). Defensive strategies of soft corals (Coelenterata: Octocorallia) of the Great Barrier Reef. II. The relationship between toxicity and feeding deterrence. Biol. Bull. mar biol. Lab., Woods Hole 171. 565-576

Lindquist, N., Fenical, W., Van Duyne, G. D. Clardy, J. (1988) New alkaloids of the lamellarin class from the marine ascidian Didemnum chartaceum (Sluiter, 1909). J. org. Chem. 53: 4570-4574

Matsunaga, S., Fusetani, N., Hashimoto, K. (1986). Bioactive marine metabolites. VIII. Isolation of an antimicrobial blue pigment from the bryozoan Bugula dentata. Experientia 42: 84

Millar, R. H. (1971). The biology of ascidians. Adv. mar. Biol. 9: $1-100$

Morris, R. H., Abbott, D. P., Haderlie, E. C. (1980). Intertidal invertebrates of California. Stanford University Press, Stanford, p. 177-226

Myers, R. F. (1983). The comparative ecology of the shallowwater species of Canthigaster (Family Tetraodontidae) of Guam. M.S. thesis, Univ. of Guam

Myers, R. F. (1989). Micronesian reef fishes. Coral Graphics, Territory of Guam

Olson, R. A. (1983). Ascidian-Prochloron symbiosis: the role of larval photoadaptations in midday larval release and settlement. Biol. Bull. mar biol. Lab., Woods Hole 165: 211-240

Parry, D. L. (1984). Chemical properties of the test of ascidians in relation to predation. Mar. Ecol. Prog. Ser 17: 279-282

Paul, V. J., Van Alstyne, K. L. (1988). The use of ingested algal diterpenoids by Elysia halimeda Macnae (Opisthobranchia: Ascoglossa) as antipredator defenses. J. exp. mar. Biol. Ecol. 119: 15-29

Pawlik, J. R., Albizati, K. F., Faulkner, D. J. (1986). Evidence of a defensive role for limatulone, a novel triterpene from the intertidal limpet Collisella Limatula. Mar. Ecol. Prog. Ser. 30: $251-260$

Pawlik, J. R., Burch, M. T., Fenical, W. (1987). Patterns of chemical defense among Caribbean gorgonian corals: a preliminary survey. J. exp. mar. Biol. Ecol. 108: 55-66

Pawlik, J. R., Kernan, M. R., Molinski, T. F., Harper, M. K., Faulkner, D. J. (1988). Defensive chemicals of the Spanish dancer nudibranch, Hexabranchus sanguineus, and its egg ribbons: macrolides derived from a sponge diet. J. exp. mar Biol. Ecol. 119: 99-109 
Randall, J. E., Hartman, W. D. (1968). Sponge feeding fishes of the West Indies. Mar Biol. 1. 216-225

Stoecker, D. (1978). Resistance of a tunicate to fouling. Biol. Bull. mar biol. Lab., Woods Hole 155: 615-626

Stoecker, D. (1980a). Distribution of acid and vanadium in Rhopalaea birkelandi Tokioka. J. exp. mar. Biol. Ecol. 48 : $277-281$

Stoecker, D. (1980b). Relationships between chemical defense and ecology in benthic ascidians. Mar Ecol. Prog. Ser 3: $257-265$

Stoecker, D. (1980c). Chemical defenses of ascidians against predators. Ecology 61: 1327-1334

Thompson, J. E., Walker, R. P., Faulkner, D. J. (1985). Screening and bioassays for biologically-active substances from forty marine sponges species from San Diego, California, USA. Mar. Biol. 88: 11-21

This article was submitted to the editor
Thompson, J. E., Walker, R. P., Wratten, S. J., Faulkner, D. J. (1982). A chemical defense mechanism for the nudibranch Cadlina luteomarginata. Tetrahedron 38: 1865-1873

Van Alstyne, K. L., Paul, V. J. (in press). The role of secondary metabolites in marine ecological interactions. Proc. 6th Internat. Coral Reet Symp

Wylie, C. R., Paul, V J. (1989). Chemical defenses in three species of Sinularia (Coelenterata, Alcyonacea) effects against generalist predators and the butterflyfish Chaetodon unimaculatus Bloch (Perciformes). J. exp. mar. Biol. Ecol. 129: 141-160

Young, C. M., Bingham, B. L. (1987). Chemical defense and aposematic coloration in larvae of the ascidian Ecteinascidia turbinata. Mar. Biol. 96: 539-544

Zar, J. H. (1984). Biostatistical analysis. Prentice-Hall, Inc., Englewood Cliffs, p. 150-155

Manuscript first received: May 31, 1989

Revised version accepted: August 29, 1989 